\title{
loT (Internet of Things) and DfPL (Device-free Passive Localisation) in a disaster management scenario
}

Deak, G., Curran, K., Condell, J., Asimakopoulou, E., \& Bessis, N. (2013). loT (Internet of Things) and DfPL (Device-free Passive Localisation) in a disaster management scenario. Simulation Modelling Practice and Theory, 34(3), 86-96. https://doi.org/10.1016/j.simpat.2013.03.005

Link to publication record in Ulster University Research Portal

Published in:

Simulation Modelling Practice and Theory

Publication Status:

Published (in print/issue): 01/02/2013

DOI:

10.1016/j.simpat.2013.03.005

\section{Document Version}

Author Accepted version

\section{General rights}

Copyright for the publications made accessible via Ulster University's Research Portal is retained by the author(s) and / or other copyright owners and it is a condition of accessing these publications that users recognise and abide by the legal requirements associated with these rights.

\section{Take down policy}

The Research Portal is Ulster University's institutional repository that provides access to Ulster's research outputs. Every effort has been made to ensure that content in the Research Portal does not infringe any person's rights, or applicable UK laws. If you discover content in the Research Portal that you believe breaches copyright or violates any law, please contact pure-support@ulster.ac.uk. 


\title{
IoTs (Internet of Things) and DfPL (Device-free Passive Localisation) in a disaster management scenario
}

\author{
Gabriel Deak $^{\mathrm{a}, 1}$, Kevin Curran ${ }^{\mathrm{a}, *, 1}$, Joan Condell ${ }^{\mathrm{a}, 1}$, Eleana Asimakopoulou ${ }^{\mathrm{b}, 2}$, Nik Bessis ${ }^{\mathrm{b}, 2}$ \\ a Intelligent Systems Research Centre, University of Ulster, Derry BT48 7JL, UK \\ ${ }^{\mathrm{b}}$ Distributed and Intelligent Systems (DISYS) Research Group, School of Computing and Mathematics, University of Derby, Kedleston Road, Derby, DE22 1GB, UK
}

\section{A R T I C L E I N F O}

\section{Article history:}

Received 1 August 2012

Received in revised form 11 February 2013

Accepted 15 March 2013

\section{Keywords:}

Localisation systems

Wireless sensor networks

Device-free passive localisation

Internet of things

Disaster management systems

\begin{abstract}
A B S T R A C T
This paper presents an overview of the potential obstacles and challenges related to research topics such as IoT, DfPL WSNs (Wireless Sensor Networks) in IoT and disaster management using WSNs. This review will analyse key aspects of deploying a DfPL WSN in IoT scenario for disaster management.

In an IoT scenario the DfPL WSN is only collecting raw data that is forwarded to the Internet using a Compressed Sensing (CS) IoT framework or other solutions including data compression. Compressed Sensing (CS) refers to a method used to reduce the number of samples collected in an IoT WSN. Thus it is possible to create stand-alone applications that require fewer resources. There is no need to process the data in the WSN as this can be done in the Data Analysis Network, after the data is reconstructed. This will enable a reduced volume of data transmitted and lower power consumption for battery-operated nodes.

The detection of people in a disaster scenario who are simply moving and not in the possession of a 'tracking device' is revolutionary. The aim here is to build upon our patentpended technology in order to deliver a robust field-trial ready human detection system for disaster situations.
\end{abstract}

(c) 2013 Elsevier B.V. All rights reserved.

\section{Introduction}

The Internet of Things (IoTs) is an emerging paradigm shaping our current understanding about the future of Internet where we transform physical object to smart objects which can be interlinked through IP. IoT has been emerged as one of the most important paradigmatic strings of thought with regards to the future state of Internet. Its significance is described in terms of providing a different lens on how to link the Internet with real world's objects. In a more comprehensive way, IoT transforms real world objects into smart objects and connect them through Internet. In contrast with current Internet, IoT depends on a dynamic architecture where physical objects with embedded sensors will communicate with an einfrastructure (i.e. a cloud) to send and analyse data using the Internet Protocol. IoT envisions a future in which digital and physical entities can be linked, through their unique identifier and by means of appropriate information and communication technologies. There are several works highlighting notable challenges for creating a framework to enable interoperability between resources (or objects) as part of an enlarged future enterprise [43-45].

\footnotetext{
* Corresponding author. Tel.: +44 (0)28 71375418; fax: +44 (0)28 71375470.

E-mail address: kj.curran@ulster.ac.uk (K. Curran).

1 School of Computing and Intelligent Systems, Faculty of Computing and Engineering, University of Ulster, Derry BT48 7JL, UK.

2 School of Computing and Mathematics, University of Derby, Kedleston Road, DE22 1GB, UK.
} 
A notable challenge is the estimation of the positioning of an object either indoors or outdoors. The number of applications using location information is growing and the expectation is to expand significantly when IoT objects will require to purposefully get connected. Various indoor location-aware systems have been developed using metrics such as RSSI (Received Signal Strength Indicator), TOA (Time of Arrival), TDOA (Time Difference of Arrival) and AOA (Angle of Arrival). The majority of these systems are active systems where the tracked object (i.e. a person) is participating actively which means the person is carrying an electronic device sending information necessary to estimate the location. Indoor Device-free Passive Localisation (DfPL) technology uses an RSSI-based method to record variances of a measured signal where an IoT person being tracked is not carrying any electronic device that can be used to estimate the location. The system monitors the changes in the RSSI measurements caused by the presence of a human body in an indoor environment. For example, it is known that the resonance frequency of water is $2.4 \mathrm{GHz}$ and the human body contains more than $70 \%$ water. Thus the human body attenuates the wireless signal reacting as an absorber. Refs. [14-16] present various features of DfPL systems. The features can be classified as follows: tracking, identification, single person detection, multi-person detection and automatic construction of a passive radio map. The drawback of using RSSI measurements is the fact that this type of measurements is very noisy. In our approach we evaluate various smoothing algorithms and the results show an improvement in detecting the presence of the person indoors. RSSI measurements are recorded in a Wireless Sensor Network (WSN) deployed using Java Sunspot nodes and a Base Station (Java Sunspot Development kits). IoT empowers every object around us with computational and communication capabilities. This can be achieved by attaching devices or sensors to objects. The network using Radio Frequency Identification (RFID), infrared sensors, Global Positioning Systems (GPS), and any other information sensing technologies embedded in things or everyday items is defined as IoT [22]. Wireless Sensor Networks (WSNs) are viable solutions as the objects can communicate and exchange data with people or other objects.

We outline here the use of IoT DfPL for the management of disasters in first response emergency scenarios. The IoT objects are equipped with Java Sunspot Nodes deploying a DfPL WSN. Data collected can be accessed and used by first response teams in the case of an emergency or disaster. Our focus is to analyse the possibility of detecting multi-occupancy in the monitored environment. Detecting human presence without the possibility of counting the number of people inside a space can be helpful for the emergency team. The aim is to demonstrate the use of a DfPL WSN in an IoT scenario based on existent protocols for emergencies or disaster situations management In an IoT scenario the DfPL WSN is only collecting raw data that is forwarded to the Internet using a CS IoT framework or other solutions including data compression. There is no need to process the data in the WSN as this can be done in the Data Analysis Network, after the data is reconstructed. This will enable a reduced volume of data transmitted and lower power consumption for battery-operated nodes. The detection of people in a disaster scenario who are simply moving and not in the possession of a 'tracking device' is revolutionary. The aim here is to build upon our patent-pended technology in order to deliver a robust field-trial ready human detection system for disaster situations.

The remainder of this paper is organised as follows: Section provides an overview of device free passive localisation, Section 3 describe non-human factors in wireless signals, Section 4 describes WSN in IoT, while Section 5 discusses the possibility of deploying WSN for disaster management. Section 6 presents passive localisation techniques for disaster management. Section 7 introduces the architecture of IoT DfPL and Section 8 concludes the paper.

\section{Device free Passive Localisation (DfPL)}

Various DfPL systems will be presented as an introduction to passive localisation. Various techniques such as Ultra-wideband (UWB), Physical Contact, Differential Air Pressure, Computer Vision, and Device-free Passive Localisation (DfPL) have been used in indoor passive localisation.

Ultra-wideband (UWB) is one of the first techniques used to deploy passive localisation systems [1]. Through-the-wall surveillance or through-wall imaging (TWI) techniques are used to denote UWB passive systems [2-4]. This technique has been recently used for both static and motion detection. UWB passive localisation is considered to be an extension to a technique called radio tomographic imaging due to its similarity to the medical tomographic imaging. Through-wall imaging refers to the ability of detecting and monitoring objects or people through buildings walls. This can be very useful to law enforcement agencies and can have many applications in military and civil scenarios [5]. UWB has the advantage of being able to penetrate walls. Various implementations of UWB technique have been proposed. A UWB system has the following two main components: transmitters and receivers. Short pulses are sent by a pulse generator via a horn antenna [6]. The receivers wait and monitor echoes from various objects or people. TileTrack represents a low cost two-dimensional location estimation system based on physical contact [7]. Changes in the capacitance between transmitting and receiving electrodes (plate electrodes or wire electrodes) are monitored. The system is based on nine floor tiles with one transmitting electrode for each tile. Each tile is $60 \mathrm{~cm}$ by $60 \mathrm{~cm}$ square-shaped made from thick chip-board with thin steel coating. The prototype used to deploy the TileTrack technique has a square tracking area with a size of $3 \times 3$ tiles. AirBus estimates location based on indoors airflow disruption caused by human movement [8]. An air pressure sensor is placed within the central heating, ventilation, and air conditioning (HVAC) unit. The sensor detects pressure variations. AirBus can correctly identify an open or closed door $80 \%$ of the cases with HVAC in operation and $68 \%$ with HVAC unit switched on.

Computer vision can be considered as a DfPL system because the tracked people are not carrying any electronic devices or tags. The EasyLiving project [9] is a computer vision based system which aims to transform any environment in a smart 
environment dependent on location information. Possible applications include switching on/off devices near to the users location, monitoring people's behaviour and many others. The system architecture consists of three PCs (Personal Computers) and two sets of colour cameras. Each camera is connected to one PC, while the third PC is used for running the person tracker algorithms. Video processing algorithms are used to separate and track people. The system was tested with a maximum of three people in the environment. The possibility of obstructions depends on the behaviour and the number of persons.

The Device-free Passive Localisation (DfPL) [10-13] is based on monitoring the variances of the signal strength in a wireless network. The human body contains about $70 \%$ water and it is known that waters resonance frequency is $2.4 \mathrm{GHz}$. The frequency of the most common wireless networks is $2.4 \mathrm{GHZ}$, thus the human body behaves as an absorber attenuating the wireless signal [2,5,14-17]. This technique is the focus of our research and the remainder of the paper is based on DfPL using Wireless Sensor Networks (WSNs).

\section{Non-human factors which affect wireless signals}

Errors in location estimation can be caused by many factors. Various error sources such as multipath, non-line-of-sight (NLOS), interferences and shadowing effects have been identified in [40]. These can affect RSSI localisation systems. Other error sources that were found to affect systems based on radio frequency technologies are clock synchronisation, multipath, variable atmospheric conditions, differences in the conducting and reflecting properties of materials, direction of antennas, geometry, possible attacks, unavailability of base stations. This section will discuss some of the most common source of errors in the radio frequency-based localisation systems.

NLOS is radio transmission using a path that is obstructed, usually due to a physical object and could affect a location estimation [41]. The best example here is GPS which is based on the line-of-sight (LOS) with the satellites. Permanent availability of the line-of-sight is ideal, but due to many factors this cannot be achieved. Thus, measurements errors will affect the accuracy and precision of the localisation system. However, dead-reckoning, has been used successfully with GPS and other technologies to improve the accuracy when exact measurements are temporary unavailable. Location estimation using dead reckoning is based on previous information about the location of the tracked device, direction and speed. Indoors, Wi-Fibased systems for example, could be affected by the NLOS. NLOS is usually caused by a low amplitude of the signal transmitted between two terminals or the signal is completely blocked by obstacles, known as occlusion. Due to a very low amplitude the signal cannot be differentiated from the background noise. Thus, the system's accuracy and precision will decrease.

Multipath refers to a transmitted signal that arrives at a receiver by two or more paths [42]. This is caused by the obstacle that might exist in an environment. The multipath effect can cause distortions of the amplitude and phase of the signal. In the case of NLOS, the probability of multipath increases. This is usually a common problem faced in most cases by radio waves in indoor environments. Indoors, multipath is present due to reflections, diffractions, scattering from walls, ceilings, or floor surfaces. Multipath is avoided by considering only the strongest signal that arrives at the receiver. However, this does not take into account the path of the signal, thus it is likely that the strongest signal does not arrive at the receiver on the shortest path.

If the case of two terminals with Wi-Fi capabilities, one mobile and the second one placed in a fixed location, the amplitude of the signal strength decreases with the increase of the distance between the two terminals [8]. Signal strength attenuation has been used as a technique to estimate distance between two terminals. However, indoors, signal strength measurements used with other location estimation techniques might be affected due to signal attenuation caused by obstacles such as walls, furniture, or human presence. Attenuation is one of the main reasons of NLOS where LOS is a requirement. In wireless communications, the concept of fading represents a deviation of the attenuation. It can be caused by multipath propagation or by shadowing from various obstacles. Both scattering and reflection occur and overlap. The main factor that differentiates them is the size of the particle. Scattering appears for particles smaller than the light wavelength, and reflection for larger particles.

\section{WSN and IoT}

IoT offers the possibility of connecting trillion of things through the Internet using a single open standard interface such as IP (Internet Protocol). Internet of Things (IoTs) also known as Web of Things (WoT) is a concept where everyday devices home appliances, sensors, monitoring devices - can be accessed through the internet using well known technologies such as URLs and HTTP requests [18,19]. There are still open issues regarding five IP-WSN (Internet Protocol Wireless Sensor Network) features in an IoT scenario: IPv6 Adaptation, Mobility, WEB Enablement, Time Synchronisation and Security. Ref. [20] presents SNAIL (Sensor Networks for an All-IP World) which integrates four important features: mobility, web development, time synchronisation and security with a complete IP architecture adapted to WSN. The standard dedicated IP stack needs to be adapted to low-processing-power microprocessors such as the ones found in WSNs. Mobility is one important characteristic of a WSN. A protocol that supports high speed communication on nodes and route optimisation is required. WEB Enablement offers simple access to things using a web browser. Global time synchronisation between things is necessary if the things are in different parts of the world. In the WSNs case the security needs to be both lightweight and robust. 
MARIO (Mobility Management Protocol to Support Intra-PAN and Inter-PAN Handover with Route Optimisation for 6LoWPAN) is a mobility management protocol that was introduced in [20]. Two cases are shown. Firstly, when movement of Mobile Node ( $\mathrm{MN})$ is detected a partner node preconfigures a future handover. In the second case, the partner node that detects MNs movement it sends a preconfiguration message with all the information about MN to a candidate foreign agent (FA) which stores the details of MN. WSN are considered as one of the key elements of IoT due to the possibility of embedding mobile transceivers into everyday items. This enables a new form of communication where people can interact with things and things can interact with each other. However, WSN technology is still under research. Many researchers focus on routing protocols, network architecture, access protocols and indoor channel models [21]. Any improvement means an increase in communication speed, reliability and security of WSNs in IoT scenarios.

Ref. [22] discusses the complexity of IoT referring to characteristics of complex networks. The later structure of IoT is composed of sensing layer, transport layer and application layer. Various network architectures are discussed such as independent network, hybrid network and access point network. An independent network uses a single gateway between a WSN and Internet. The drawback is the possibility of a gateway failure which will disconnect the WSN from the Internet. The second and third architecture ensure a more robust network and the choice between them depends on the application requirements. In the hybrid network case a number of selected nodes have access to the Internet and behave as gateways. The last architecture is inspired from the current WLAN (Wireless Area Local Network) where nodes can access Internet in one hop through multiple gateways.

Ref. [23] presents an IoT Gateway prototype based on Zigbee and GPRS (General Packet Radio Service) protocols. Currently the DfPL WSN is deployed as an ad hoc network with no connection to the Internet. Thus there is a need to develop the communication protocols between the WSN and the Internet, to convert different sensor network protocols and to verify the functionality of the entire system. A key aspect of using IoT Gateway is to identify the necessary elements to inter-connect different sensor networks with traditional communication networks. Three layers are required in order to deploy applications using an IoT WSN. These are the perception layer, transmission layer and application layer. The perception layer contains WSN DfPL nodes which collect signal strength measurements from the interlinked objects. In the transmission layer the system aims to send the data using traditional broadband networks, WiFi or any other means of communication over longer distances. The application layer runs data processing algorithms and services dependent on application requirements.

An IoT Gateway system requires data forwarding, protocol conversion, management and control, and software. Data forwarding is necessary in order to correctly transfer data from WSNs to other communication networks. Thus, the conversion between various protocols must be done. An IoT system should also offer management and control over the WSN. Management and control functions could be used to change various system parameters. In order to take advantage of an IoT Gateway system, personalised software for each component and layer must be developed. Many different sensors and technologies both wired and wireless have already been deployed over the years. Deploying new network infrastructures from scratch would be difficult [24], thus a very important challenge is to develop a framework that can integrate existent networks with new infrastructures. An IoT Gateway system can cope with direct communication between different standards through the protocol conversion module. However the current trend is to use an all-IP infrastructure that will provide any network infrastructure with connectivity to the Internet. Thus this will allow the interconnection of smart objects with a network interface in an IoT infrastructure where every device has its own IP. Other challenges in IoT scenario are the scalability, privacy, security, big volumes of data, fault tolerance and in the case of battery-operated objects, the power consumption.

The IoT WSN needs to be scalable to a large number of objects and people. Privacy and security are necessary for applications that use billing information for example. Even though the amount of data exchanged by objects through the embedded sensors is not large, over all due to the large number of objects and the frequency of transmission the infrastructure needs to deal with big volumes of data. Another problem in an IoT scenario is the need of redundancy in several levels and automatic adaptation to changes in order to achieve a robust communication. A large number of smart objects are battery-operated, thus a critical aspect in this case is the power consumption. Ref. [24] presents an overview of existent solutions for deploying WSNs in an IoT scenario. There are Non-IP solutions such as Zigbee, Z-Wave, INSTEON and Waveins, IPbased solutions using IPv6 without the need of gateways or proxies, and high level and middleware solutions.

Data compression ratio, a very important aspect in IoT, is discussed in [25]. Due to the large number of sensors, an IoT scenario deals with big volumes of data. There are three main problems that must be solved: resolution, sensitivity, and reliability. Compressed Sensing (CS) refers to the method used to reduce the number of samples collected in an IoT WSN. Thus it is possible to create stand-alone applications that require fewer resources. A CS framework using a cluster-sparse reconstruction algorithm focuses on network data compression with an accurate data reconstruction and a long network life [6]. The CS IoT framework contains three components: Data Acquisition Networks, Internet Network and Data Analysis Network. The Data Acquisition Network manages collection, compression and forwarding the data to the Internet Network, while the Data Analysis Network reconstructs the data with minimum distortions.

\section{Disaster management using WSN}

CodeBlue represents a software infrastructure that addresses existent challenges in WSN deployment [26]. CodeBlue integrates wireless sensor nodes and other devices with a wireless network interface (e.g., wireless vital sing monitors) into a system for disaster management which can provide valuable information to emergency responders. Deploying and testing 
such an infrastructure presents some specific challenges such as: discovery and naming, robust routing, prioritisation of critical data, security and tracking device location. The solutions to cope with these issues are explained in more detail in [26]. The system has a flexible naming scheme, robust routing framework, authentication and encryption, connection establishment and handoff, RF-based location tracking, filtering and aggregation modules.

Ref. [27] analyses the usage of WSN in order to secure important infrastructures and manage disasters in USA based on the features defined by The National Security Telecommunications Security Convergence Task Force report for The National Security and Emergency Preparedness (NS/EP) of the USA. It is shown that WSN cannot meet all the requirements, thus a more complex network infrastructure is required. The requirements for sensor networks are: enhanced priority treatment, prevention of corruption of data or unauthorised access, service robustness, interconnection and interoperability with government or private systems, mobility, management of high capacity communication services, voice and data routing protocols, consistent and precise performance. Researchers have been focusing on WSN routing protocols that offer power consumption optimisation. In order to test and calibrate WSN offline, an independent network with its own service and security mechanisms is required. It has been found that wireless sensor networks meet the following requirements: mobility, service robustness, Web Services protocols for interconnectivity and interoperability of the sensors with other systems.

Ref. [28] uses location aware distributed sensor networks in order to deploy a natural disaster management system. The availability of location information as part of the system installation process is a necessity. This requirement is important in order to discover optimal routing paths that can achieve maximum lifetime of the system. There are many researchers that focus on the deployment of distributed sensor networks that monitor unreachable or hostile areas. The system uses a clustering approach where membership of a node is set to one or more heads based on the proximity. Each cluster head finds the shortest path to transmit data to the base station. The system also considers the case when one of the heads is not available anymore due to a power supply problem or other faults. Head rotation is the method used to balance load between heads or to appoint a new head. Both location information and clustering approach are important in order to minimise the hop count which reduces energy consumption.

Refs. [29-31] present a Wireless Sensor Network Protocol for Disaster Management (WSNPDM) and respectively analyse the performance of this protocol compared to LEACH clustering protocol. WSNPDM is a clustering algorithm developed in order to overcome deficiencies in communication between rescuers and survivors in a disaster scenario. Current communication systems can cope with disaster conditions as long as the communication is not completely interrupted. WSNPDM can be deployed for rescue purposes in the case of communications in an existent cellular network being completely interrupted. The protocol considers collapsed base stations and can convey data to the nearest base station available. They use the following as performance factors: average energy dissipation, system lifetime, data delivery rate and number of live nodes.

\section{Passive localisation techniques in disaster management}

Ref. [32] discusses perception techniques for fire scenarios, urban or industrial. The system detects smoke and tracks persons using cameras. It is implemented based on the AWARE platform. AWARE focuses on fire detection scenarios and has the following elements: ground wireless sensor network (GSN) with temperature, humidity, smoke sensors, unmanned aerial vehicles (UAVs), and the body area network (BAN) with hearth rate, temperature, and oxygen saturation monitors [33]. Ref. [32] uses computer vision with infrared detection techniques but it is difficult to detect smoke as smoke does not have necessary features to be differentiated from background in all cases. Techniques such as colour detection, motion detection, and texture detection have been used to detect smoke. The outputs of these techniques are later combined to obtain a more accurate smoke detection. Firemen detection is obtained by applying colour segmentation and blob segmentation.

Refs. [34,35] introduce an Ultra-wideband (UWB) system based on an ad hoc network that enables location estimation of the emergency personnel in emergency or disaster scenarios. Many other systems such as infrared, ultrasound, or WLAN require pre-installation. In the case of a disaster the infrastructure used by these systems might be destroyed, inoperable or inexistent. However, the previous section discussed viable WSNs solutions for emergency and disaster situations.

The UWB system proposed in [34,35] has four key elements: a control centre, anchor terminals, mobile terminals and dropped terminals. Mobile terminals are carried by the personnel and communicate with anchor terminals directly, or using dropped terminals in the case where the signal strengths are low. The location of the mobile nodes is obtained using the triangulation technique based on at least four reference nodes. The mobile terminals include telemetry sensors and an alarm button. The anchor nodes are generally installed on emergency vehicles, are equipped with differential GPS, and are used as reference nodes for the mobile nodes. After the location of a mobile node has been obtained the mobile node also becomes a reference node. WLAN communication is used in order to avoid congestions or to increase the speed of the radio links. Interferences between transmission and location estimation are reduced by using synchronised time slots. One very important aspect and future work is the design of routing algorithms. Due to limited data bandwidth, traditional routing protocols are not suitable for the proposed system. The system uses an anycast routing protocol having the routing information included in the packets header.

To the best of our knowledge, IoT or/and disaster management implementations for other localisation systems presented in Section 1 such as Physical Contact, Differential Air Pressure, and Device-free Passive Localisation (DfPL) have not been developed. 


\section{DfPL in IoT architecture}

This project focuses on deploying a DfPL system on top of a Wireless Sensor Network (WSN). The first step towards implementing such a system is filtering data using a selected smoothing algorithm, SavitzkyGolay smoothing filter in this case. The filtered data is fed to traditional classifiers (e.g. Naive Bayes or TreeBagger) in order to detect people's presence. The next step is using classified data, timestamps and links affected as parameters in a decision making algorithm that will detect the presence of people in the monitored environment. Such an environment is presented in Fig. 1. Four Java Sunspot nodes were placed randomly in a room of size $3.6 \mathrm{~m}$ by $3.4 \mathrm{~m}$. Fig. 2 shows the effect that a person has upon the wireless communication.

In the case of four nodes we collect data from 12 links as each node in the network uses a bidirectional connection with every other neighbour node. Multiple collection threads, more than one base station, or transmission on multiple channels improve the collection speed in case of congestions.

Fig. 3 shows the architecture of an IoT DfPL system which records RSSI measurements that can be used to detect if people are present in an environment. A protocol to parse, manipulate and process HTTP requests is implemented on a web server that uses traditional communication protocols such as TCP or UDP. This protocol works even for Reverse-HTTP (RHTTP) [36]. Thus, HTTP requests can be handled even if the protocol is running behind a firewall/NAT and data is available via a RHTTP gateway. There is a demo implementation of a WoT included in the Sunspot Development Software Development Kit (SDK). Sensors from the Sunspot nodes can be accessed from anywhere on the internet by simply using a URL similar to the one in Fig. 3.

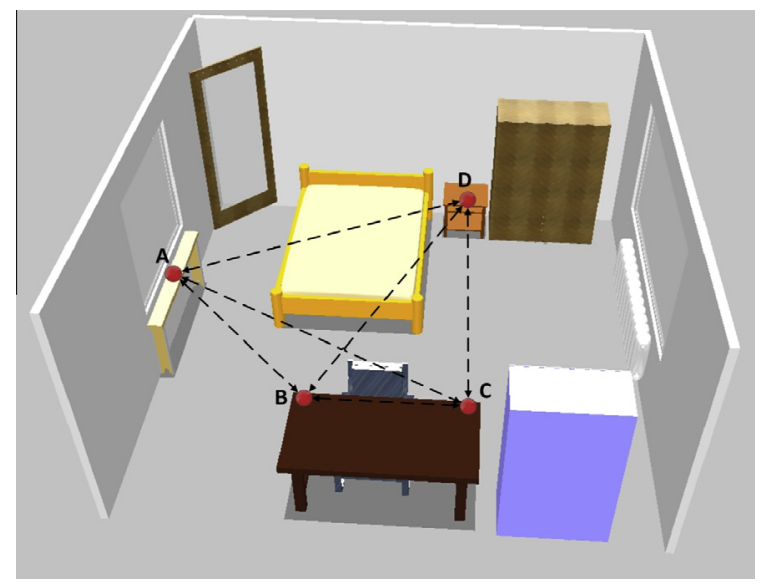

Fig. 1. DfPL test bed with four nodes in random locations in a room.

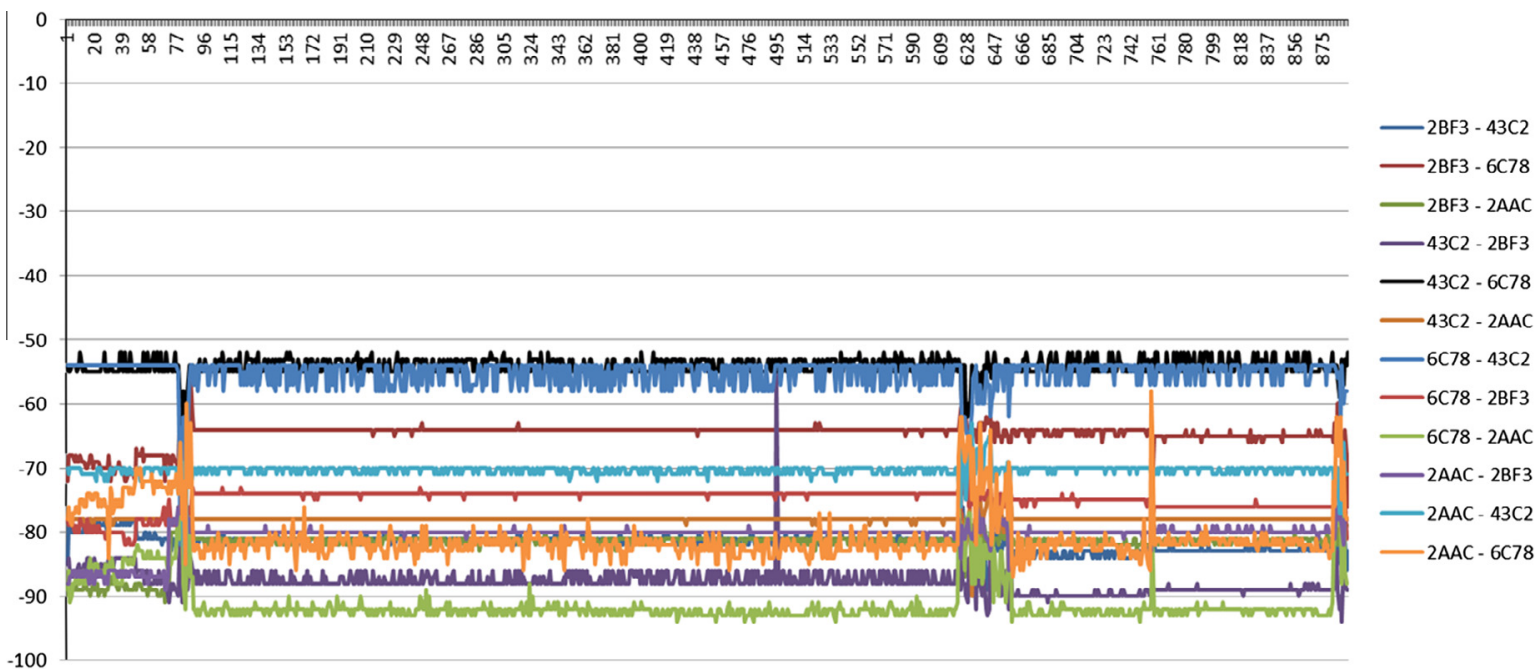

Fig. 2. RSSI measurements from a test bed with four nodes. 


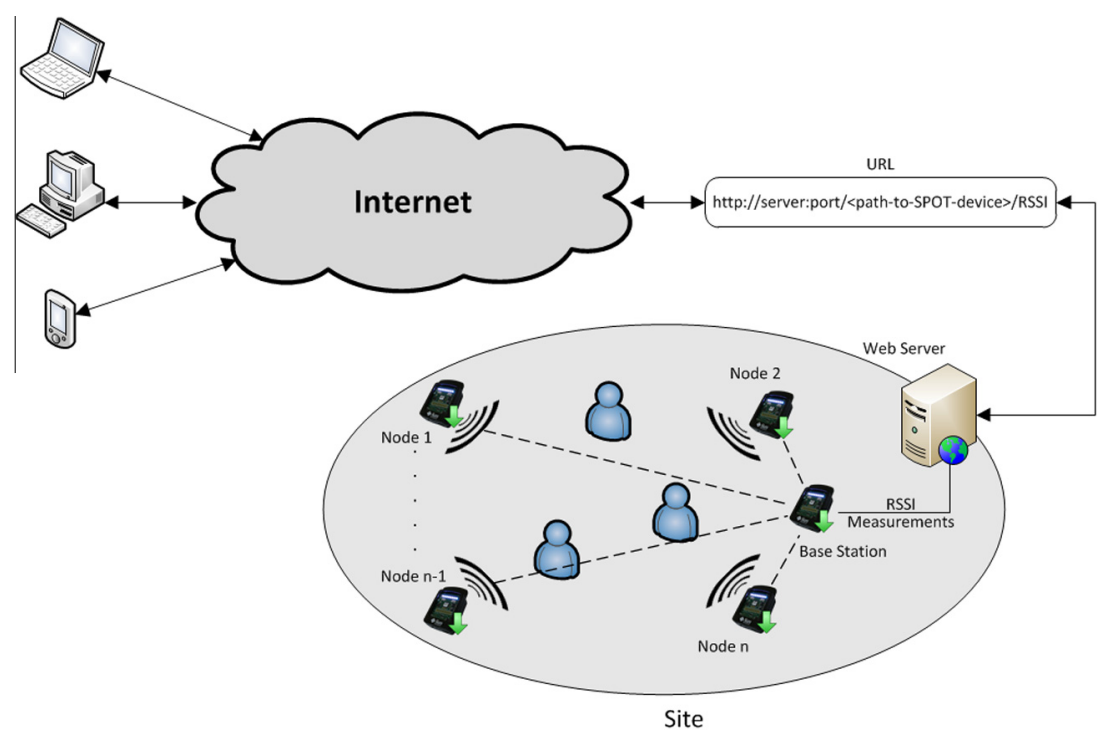

Fig. 3. IoT DfPL architecture.

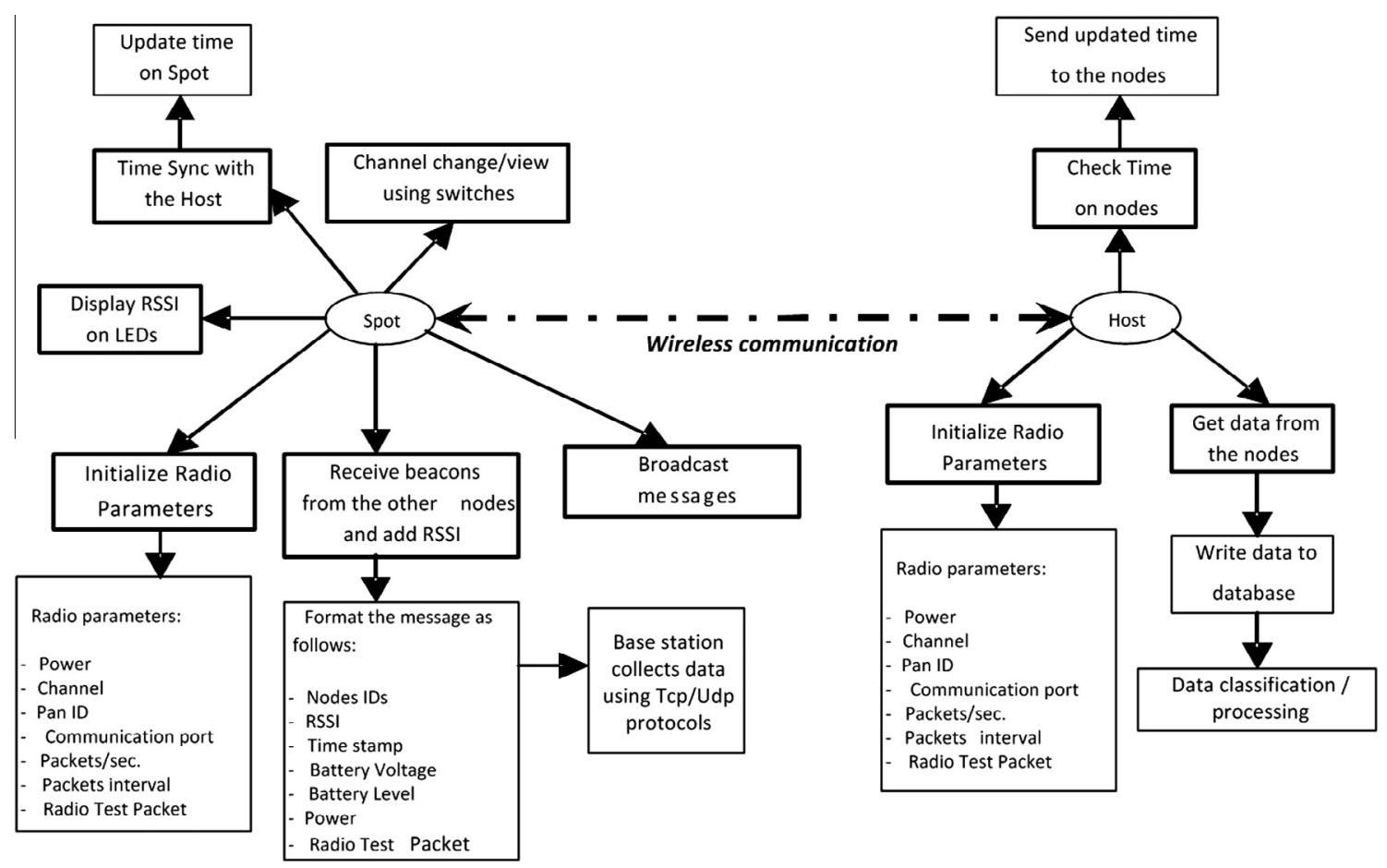

Fig. 4. WSN functions implemented on spots and host.

The site is monitored by a WSN with $n$ Java Sunspot nodes depending on its size. RSSI measurements are recorded between nodes, forwarded to the base station and stored on a server. Recorded data is stored in a database on the server and can be synchronised in the cloud. This is a viable solution for a framework used in disaster or emergency situations. The process of detecting people in the environment is running on the server or on the device requesting data from the system. The Wireless Sensor Network (WSN) is based on four IEEE 802.14.5 Java Sun SPOT nodes and a base station deployed in the environment. Fig. 4 shows all the function currently running within the proposed WSN. Time synchronisation between the spots and host is very important. Timestamps are used in order to detect when an event has occurred and identify all the links affected by human presence. Without time synchronisation this will not be possible. The signal strength is also 
displayed using the LEDs on each spot. However, after the time is synchronised, it is important to initialise radio parameters required for communication. The communication channel and transmission power can be changed using the existent switches. Each spot broadcasts messages and in the same time listens for other nodes broadcasting. Once a packet has been received, the receiving node formats a message by adding the following fields: address, RSSI, timestamps, battery status, and radio parameters. The data collection is controlled by the base station. The base station selects the node to be read and stores all data in a database.

Classifiers (see Fig. 7) are used for motion detection [37]. Other approaches, such as Moving Average Based Detection or Moving Variance Based Detection, can be used depending on the device processing recorded data [15]. The Naive Bayes classifier has two steps: training and prediction [38]. Similar to other classification methods in the training phase, Naive Bayes uses training vectors, pairs of inputs-outputs, to estimate the parameters of probability distributions. The prediction step uses unseen data and computes the posterior probabilities based on the parameters obtained in the training phase. The posterior probabilities are used to classify inputs belonging to each class.

The independence assumptions allow the classifier to compute the parameters for an accurate classification using smaller training samples compared to other classifiers. This has been shown to work even for features which are not independent of one another. The Naive Bayes classifier gives the possibility of using various distributions depending on features that needs to be identified. The following distributions are supported: normal (Gaussian), kernel and multinomial distributions. The input vectors we are classifying follow a normal (Gaussian) distribution.

The probability model for a classifier is given by:

$$
p\left(C \mid F_{1}, \ldots, F_{n}\right)
$$

where $C$ represents an dependent class and $F_{1}, \ldots, F_{n}$ are the features variables. The Naive Bayes classifier implements Bayes theorem given by:

$$
p\left(C \mid F_{1}, \ldots, F_{n}\right)=\frac{p(C) p\left(F_{1}, \ldots, F_{n} \mid C\right)}{p\left(F_{1}, \ldots, F_{n}\right)}
$$

which means that the posterior probabilities are computed as follows:

$$
\text { posterior }=\frac{\text { prior } \times \text { likelihood }}{\text { evidence }}
$$

One can notice that the denominator does not depend on the class variable $\mathrm{C}$, thus it is constant as the features values are known. In practice we only need to consider the numerator of Eq. (3) which is equivalent to the joint probability model expressed by:

$$
p\left(C ; F_{1}, \ldots, F_{n}\right)
$$

The naive conditional assumptions can be used. If we consider that feature $F_{i}$ is independent of every other feature $F_{j}$ where $j, i$ we have:

$$
p\left(F_{i} \mid C ; F_{j}\right)=p\left(F_{i} \mid C\right)
$$

Based on Eq. (5) the joint probability model can be written:

$$
p\left(C, F_{1}, \ldots, F_{n}\right) \propto p(C) p\left(F_{1} \mid C\right) p\left(F_{2} \mid C\right) p\left(F_{3} \mid C\right) \ldots \propto p(C) \prod_{i=1}^{n} p\left(F_{i} \mid C\right)
$$

Based on Eq. (6) we can now rewrite conditional distribution over the class variable $C$ which can be expressed as:

$$
p\left(C \mid F_{1}, \ldots, F_{n}\right)=\frac{1}{p\left(F_{1}, \ldots, F_{n}\right)} p(C) \prod_{i=1}^{n} p\left(F_{i} \mid C\right)
$$

The RSSI data to be classified is a continuous input vector $\mathbf{X}$ with a Gaussian distribution. The input vector is segmented into classes, and then we compute the mean and the variance of $\mathbf{X}$ in each class $\mathrm{C}$. The mean value of attribute values $X_{j}$ for which $C=c_{i}$ is denoted by $\sigma_{j i}$ and the variance (standard deviation) of the inputs $X_{j}$ for which $C=c_{i}$ is denoted by $\sigma_{j i}^{2}$. The probabilities for the values in the input vector are computed as follows [39]:

$$
p\left(X_{j} \mid C=c_{i}\right)=\frac{1}{\sqrt{2 \pi \sigma_{j i}^{2}}} e^{-\frac{x_{j}-\mu_{j i}}{2 \sigma_{j i}^{2}}}
$$

We have selected one bidirectional link between nodes A and B as shown in Fig. 1. Both links are considered to be independent. Fig. 5 shows the raw data collected from the selected bidirectional link. Each data set contains 800 values.

The first data set represents the training data while the second one is the test data. The nodes are broadcasting messages every $200 \mathrm{~ms}$. When the messages are received, Received Signal Strength Indicator (RSSI) is added and then the messages are forwarded to the base station. Recorded data sets are smoothed in order to filter noise. The derivative of the signal normalises the data. Fig. 6 shows the smoothing, derivative and threshold selection on one of the links. Data from the second link is 

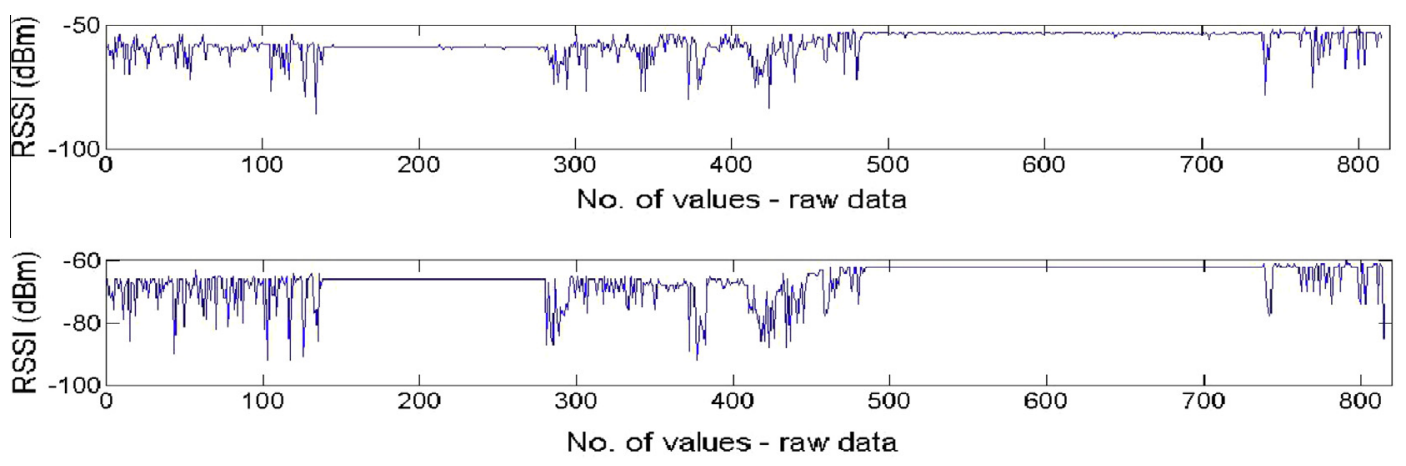

Fig. 5. Raw data from bidirectional link.
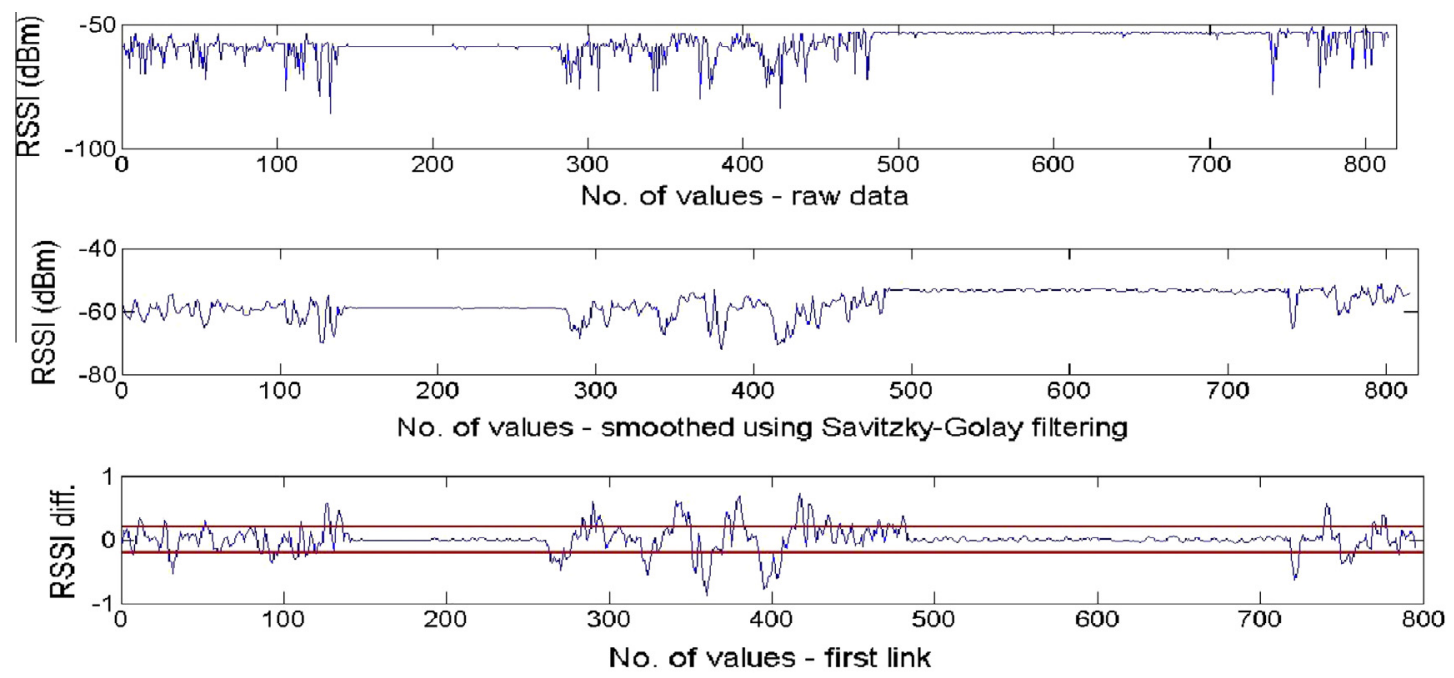

Fig. 6. RSSI processing.
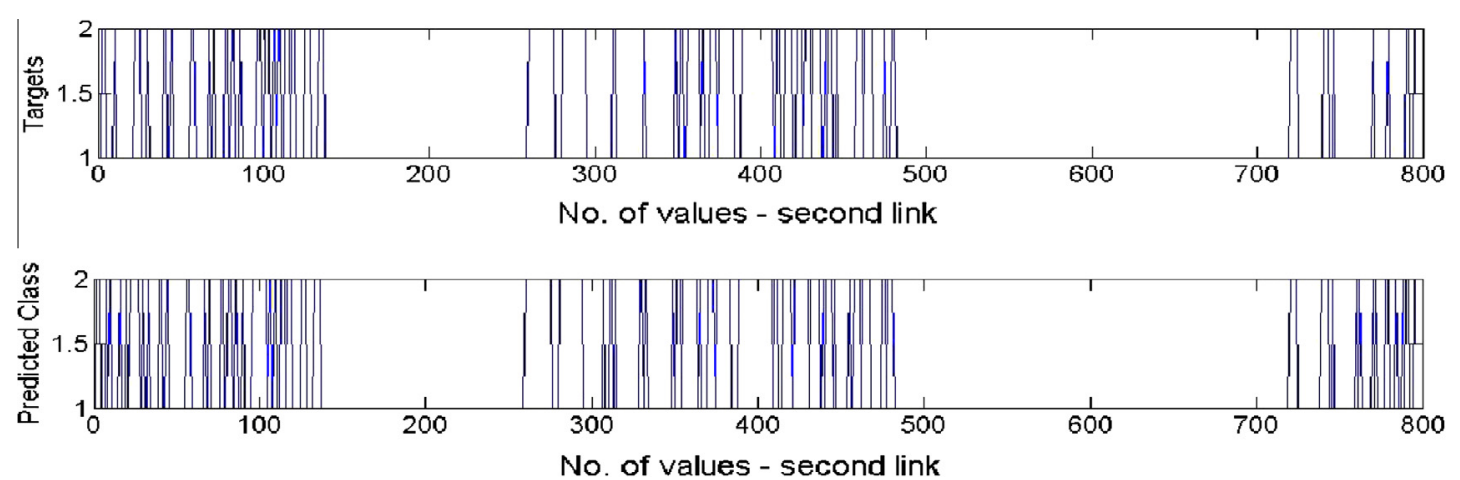

Fig. 7. Targets and predicted classes using Naive Bayes with normal (Gaussian) distribution.

processed in a similar manner. It is necessary to normalise the data in order to train and use classifiers. The threshold selection considering the normalised data in this case was 0:2. Any other value above or below this threshold is considered an event which will be classified as motion. The threshold is dependent on the environment.

In very noisy environments we need to modify this threshold. Thus a calibration depending on the level of noise in the environment is required. Thus, the calibration process can be considered as a drawback in a disaster scenario. However, DfPL can be deployed using the proposed architecture or it could be implemented on an existent WLAN infrastructure. 
Fig. 7 represents the output from the Naive Bayes classifier with a normal (Gaussian) distribution. One link is used to train the classifiers while the data recorded on the second link represents the test vector. The targets vector is obtained by analysing the data based on the threshold chosen above. Afterwards the test data is fed to the classifier and the output is compared with the targets vector. It can be noticed the data is classified into two classes: 'No motion' (value 1) and 'Motion' (value 2). The classification output, time stamp and affected links will be used as parameters in a decision making algorithm to determine the people's presence in the monitored environment.

The results showed the possibility of using classifiers in order to detect peoples' presence in a DfPL system. Thus, we deploy a disaster management system based on the proposed IoT DfPL architecture. In the case of a disaster, recorded data is used to verify if people are present in the environment and take necessary actions. This system can be used even if the infrastructure has collapsed, due to data being stored in the cloud. Thus, if the system is completely unavailable, we can use the last stored data. In the case the system is partially affected, live measurements combined with the last complete data is used to determine if there is any person in the affected area.

\section{Conclusion}

In this paper we have presented various passive localisation systems and discussed key aspects of WSNs in IoT scenarios. There are many disaster management systems that use WSNs. However, there is no system that uses IoT (WoT) WSNs in a disaster management scenario. Therefore, we have introduced a novel disaster management system that takes advantage of WSN DfPL concept. Classifiers and approaches such as Moving Average Based Detection or Moving Variance Based Detection are used for motion detection. This is an on-going work but we are confident that the proposed concept can be beneficial in terms of disaster management or emergencies.

Currently, the proposed WSN works locally in a monitored environment. Thus, in order to deploy a WSN IoT scenario we will modify the firmware running on the spots. Further, the host PC will run as a web server that is used to access the nodes from anywhere in the world over the internet. Future work involves identifying cloud storage solutions. Cloud storage will be used to store raw data collected from proposed WSN. Cloud computing and storage will provide data in the case that the proposed WSN has completely or partially collapsed during a disaster.

\section{Acknowledgements}

We would like to acknowledge the European funded Project UNITE (FP7 248583), namely its secondment programme coordinated by UNINOVA-GRIS, that supported the development of various ideas and concepts presented in this paper. It is also a pleasure to thank those who helped and supported us. Gabriel Deak would particularly like to thank his family, supervisors, colleagues and friends for their help and guidance. This paper is dedicated in loving memory of his father and his friend Iulian Stoi.

\section{References}

[1] L. Frazier, Surveillance through walls and other opaque materials, Aerospace and Electronic Systems Magazine, IEEE 11 (2002) 6-9.

[2] L. Ma, Z. Zhang, X. Tan, A novel through-wall imaging method using ultra wideband pulse system, in: 2006 International Conference on Intelligent Information Hiding and Multimedia, 2006, pp. 147-150.

[3] J. Wilson, N. Patwari, Through-wall tracking using variance-based radio tomography networks, pp. 1-9.

[4] J. Wilson, N. Patwari, Radio tomographic imaging with wireless networks, IEEE Transactions on Mobile Computing 9 (2010) 621-632.

[5] F. Aryanfar, K. Sarabandi, Through wall imaging at microwave frequencies using space-time focusing, IEEE Antennas and Propagation Society Symposium 2004 (2004) 3063-3066.

[6] E. Gazit, Improved design of the vivaldi antenna, in: 34th Applied Imagery and Pattern Recognition Workshop (AIPR'05), vol. 135, IET, 2005 , pp. 89-92.

[7] M. Valtonen, J. Maentausta, J. Vanhala, Tiletrack: Capacitive human tracking using floor tiles, in: 2009 IEEE International Conference on Pervasive Computing and Communications, 2009, pp. 1-10.

[8] J. Krumm, Ubiquitous Computing Fundamentals, CRC Press, 2010

[9] J. Krumm, S. Harris, B. Meyers, B. Brumitt, M. Hale, S. Shafer, Multi-camera multi-person tracking for easyliving, in: Proceedings of the Third IEEE International Workshop on Visual Surveillance, IEEE, 2000, pp. 3-10.

[10] G. Deak, K. Curran, J. Condell, RSSI based device-free passive localisation, in: International European Research and Innovation Conference (IERIC 2010), 2010, p. 163.

[11] G. Deak, K. Curran, J. Condell, Wireless sensor networks - smoothing algorithms for RSSI-based device-free passive localisation, in: The Tenth International Conference on Information Technology and Telecommunications (IT\&T 2010), 2010, pp. 99-107.

[12] G. Deak, K. Curran, J. Condell, Radio communication protocols in a history aware device-free passive (dfp) localisation, in: R. Choras (Ed.), Image Processing and Communications Challenges 3, Advances in Intelligent and Soft Computing, vol. 102, Springer, Berlin/Heidelberg, 2011, pp. 469-476.

[13] G. Deak, K. Curran, J. Condell, History aware device-free passive (dfp) localisation, Image Processing \& Communications 16 (2011) 21-30.

[14] M. Moussa, M. Youssef, Smart devices for smart environments: device-free passive detection in real environments, in: International Conference on, Pervasive Computing, 2009.

[15] M. Youssef, M. Mah, A. Agrawala, Challenges: device-free passive localization for wireless environments, in: Proceedings of the 13th Annual ACM International Conference on Mobile Computing and Networking, 2007, pp. 222-229.

[16] A. E. Kosba, A. Abdelkader, M. Youssef, Analysis of a device-free passive tracking system in typical wireless environments, in: 3rd International Conference on New Technologies, Mobility and Security, 2009, pp. 1-5.

[17] L.-p. Song, C. Yu, Q.H. Liu, Through-wall imaging (twi) by radar: 2-d tomographic results and analyses, IEEE Transactions on Geoscience and Remote Sensing 43 (2005) 2793-2798.

[18] N. Bessis, E. Asimakopoulou, F. Xhafa, A next generation emerging technologies roadmap for enabling collective computational intelligence in disaster management, International Journal of Space-Based and Situated Computing 1 (2011) 76-85. 
[19] A. Zelenkauskaite, N. Bessis, S. Sotiriadis, E. Asimakopoulou, Interconnectedness of complex systems of internet of things through social network analysis for disaster management, in: Proceedings of the 4th IEEE International Conference on Intelligent Networking and Collaborative Systems (INCoS-2012), 2012, pp. 503-508.

[20] S. Hong, D. Kim, M. Ha, S. Bae, S. Park, W. Jung, J. Kim, Snail: an ip-based wireless sensor network approach to the internet of things, Wireless Communications, IEEE 17 (2010) 34-42.

[21] Y. Wang, W. Lu, H. Zhu, Experimental study on indoor channel model for wireless sensor networks and internet of things, in: 12 th IEEE International Conference on Communication Technology (ICCT), IEEE, 2010, pp. 624-627.

[22] K. Zhang, D. Han, H. Feng, Research on the complexity in internet of things, in: 2010 International Conference on Advanced Intelligence and Awareness Internet (AIAI 2010), IET, pp. 395-398.

[23] Q. Zhu, R. Wang, Q. Chen, Y. Liu, W. Qin, Iot gateway: bridging wireless sensor networks into internet of things, in: Embedded and Ubiquitous Computing (EUC), IEEE/IFIP 8th International Conference on, Ieee, 2010, pp. 347-352.

[24] L. Mainetti, L. Patrono, A. Vilei, Evolution of wireless sensor networks towards the internet of things: a survey, in: 19th International Conference on Software, Telecommunications and Computer Networks (SoftCOM), IEEE, 2011, pp. 1-6.

[25] S. Li, L. Xu, X. Wang, Compressed sensing signal and data acquisition in wireless sensor networks and internet of things, IEEE Transactions on Industrial Informatics (2012) 1.

[26] K. Lorincz, D. Malan, T. Fulford-Jones, A. Nawoj, A. Clavel, V. Shnayder, G. Mainland, M. Welsh, S. Moulton, Sensor networks for emergency response: challenges and opportunities, Pervasive Computing, IEEE 3 (2004) 16-23.

[27] R. Liscano, E. Sadok, E. Petriu, Mobile wireless rsa overlay network as critical infrastructure for national security, in: (IMS 2005 ) Proceedings of the 2005 IEEE International Workshop on Measurement Systems for Homeland Security, Contraband Detection and Personal Safety Workshop, IEEE, 2005, pp. 96-102.

[28] G. Ranjan, A. Kumar, A natural disasters management system based on location aware distributed sensor networks, in: IEEE International Conference on Mobile Adhoc and Sensor Systems Conference, IEEE, 2005, pp. 3-12.

[29] S. Sana, M. Matsumoto, A wireless sensor network protocol for disaster management, in: Information, Decision and Control, IDC'07, IEEE, 2007, pp. 209-213.

[30] S. Saha, M. Matsumoto, Performance analysis of wsndm (wireless sensor network protocol for disaster management), in: ICCT'06, International Conference on Communication Technology, IEEE, 2006, pp. 1-4.

[31] S. Saha, M. Matsumoto, A framework for data collection and wireless sensor network protocol for disaster management, in: COMSWARE 2007, 2nd International Conference on Communication Systems Software and Middleware, IEEE, 2007, pp. 1-6.

[32] J. Capitan, D. Mantecon, P. Soriano, A. Ollero, Autonomous perception techniques for urban and industrial fire scenarios, in: SSRR 2007, IEEE International Workshop on Safety, Security and Rescue Robotics, IEEE, 2007, pp. 1-6.

[33] A. Erman, L. van Hoesel, P. Havinga, Aware: platform for autonomous self-deploying and operation of wireless sensor-actuator networks cooperating with aerial objects, in: Proceedings of IEEE International Workshop on Safety, Security and Rescue, Robotics, pp. 1-6.

[34] L. Xia, A. Lo, I. Niemegeers, T. Bauge, An ultra-wide band based ad hoc networking scheme for personnel tracking in emergencies, in: PervasiveHealth 2008, Second International Conference on Pervasive Computing Technologies for Healthcare, Springer, 2008 , pp. 78-81.

[35] D. Harmer, M. Russell, E. Frazer, T. Bauge, S. Ingram, N. Schmidt, B. Kull, A. Yarovoy, A. Nezirovic, L. Xia, et al., Europcom: emergency ultrawideband radio for positioning and communications, in: IEEE International Conference on Ultra-Wideband, ICUWB 2008, 2008, vol. 13 , IEEE, pp. 85-88.

[36] D. Nelson, Reverse http Connections for Device Management Outside a Firewall, US Patent 6,553,422, 2003.

[37] G. Deak, K. Curran, J. Condell, D. Deak, Motion detection using device-free passive localisation (dfpl), 2012.

[38] MathWorks, Matlab Statistics Toolbox User's Guide, 2011.

[39] K. Chen, Naive Bayes Classifier Presentation <http://www.cs.manchester.ac.uk>, 2012.

[40] S. Ahson, M. Ilyas, Location-Based Services Handbook: Applications, Technologies, and Security, CRC Press, Inc., 2010.

[41] A. Bensky, Wireless Positioning Technologies and Applications, Artech House, 2007.

[42] K. Kolodziej, Local Positioning Systems: LBS Applications and Services, CRC press, 2006.

[43] L. Atzori, A. Iera, G. Morabito, The internet of things: a survey, Computer Networks 54 (15) (2010) $2787-2805$.

[44] M. Presser, A. Gluhak, The Internet of Things: Connecting the Real World with the Digital World, EURESCOM mess@ge - The Magazine for Telecom Insiders, vol. 2, 2009.

[45] L. Tan, N. Wang, Future internet: the internet of things, in: 3rd International Conference on Advanced Computer Theory and Engineering (ICACTE), 2010, pp. V5-376-V5-380, 20-22 August 2010. 